\title{
Sulla deformazione proiettiva dei tritessuti di curve piane.
}

\author{
Memoria di Guido Vaona (a Bologna).
}

Sunto. - Un tritessuto di curve piane $\dot{e}$, in generale, proiettivamente indeformabile. Esistono però particolari tritessuti che ammettono $\infty^{h}(0 \leq h \leq 4)$ deformazioni proiettive. Il presente lavaro è essenaialmente rivolto alla determinazione di tali tritessuti per $h=2,3,4$, e allo studio delle loro deformazioni.

\section{Introduzione.}

Si chiama tritessuto di curve piane l'ente costituito da tre famiglie di curve di un piano $\pi$, tali che da ciascun punto di $\pi$ (o di una regione di esso) passi una ed una sola curva di ciascuna famiglia. Secondo la definizione introdotta da L. Muraccirini [15] (1), una corrispondenza $T$ fra due tritessuti $\Delta$ e $\Delta^{\prime}$ si dice applicabilità o deformazione proiettiva se, per cogni coppia di panti corrispondenti $P, P^{\prime}$, esiste una omografia che muta le curre di $\Delta$ passanti per $P$ in tre curve passanti per $P^{\prime}$ ed aventi ivi un contatto geometrico del $2^{\circ}$ ordine con le corrispondenti curve di $\Delta^{\prime}$.

Segue da tale definizione che la corrispondenza $T$ è una deformazione proiettiva per $\Delta, \Delta^{\prime}$ se, e solo se, $\Delta$ e $\Delta^{\prime}$ sono i tritessuti caratteristici di $T$.

Da ciò deriva l'importanza della teoria della deformazione dei tritessuti per i riflessi evidenti che essa ha con la teoria delle trasformazioni puntuali fra piani. Mi sono proposto la presente ricerca proprio per apportare un contributo alla teoria dell'applicabilità proiettiva delle trasformazioni puntuali, recentemente introdotta da M. VILLA e L. Muracchini [19], la quale si identifica sostanzialmente con la teoria della deformazione dei tritessuti. Ma di tali applicazioni dirò in altro lavoro $\left({ }^{2}\right)$.

I tritessuti di curve piane sono stati diffusamente studiati dal punto di vista della topologia differenziale da vari Autori, fra i quali ricordo: W. BLAschke [1], G. BoL [2], J. Dubourdied [6]. Nel campo proiettivo-differenziale, in cui qui ei porremo, ricordo i lavori di E. Kollwitz [13], E. Bompiani [4], L. Muracchini [14], [15].

Allo stesso argomento che è oggetto di questo lavoro sono sostanzialmente

(1) Indichiamo con numeri fra parentesi quadre i lavori corrispondenti a tali numeri, citati nella bibliografia posta alla fine del lavoro.

(2) Alcune di queste applicazioni sono state fatte in [16] da L. Muracchisi al quale comunicai oralmente parte dei risultati contenuti in questo lavoro. 
dedicati i lavori [14] e [15] di L. MuraconINI. In tali lavori, soprattutto in [14], vengono ottenuti alcuni primi risultati, riottenuti qui con metodo diverso, dei quali diro fra poco.

Fra i problemi relativi alla teoria della deformazione dei tritessuti vi sono i problemi analoghi a quelli che si pongono nella classica teoria della deformazione proiettiva delle superficie. Va tuttavia rilevato che si presentano qui difficoltà assai più gravi. Uno dei motivi di queste maggiori difficoltà risiede nel fatto che le equazioni differenziali che reggono questi problemi non sono lineari a differenza di quanto avviene invece nella teoria delle superficie.

Poichè un generico tritessuto non è proiettivamente deformabile, il primo problema che si presenta è quello di determinare i tritessuti proiettivamente deformabili. A questa domanda è offerta risposta, esauriente dal punto di vista geometrico, dall' osservazione sopra ricordata, secondo la quale, $i$ tritessuti proieltivamonte deformabili sono $i$ tritessuti caratteristici delle trasformazioni puntuali.

Una seconda questione che può porsi è la seguente: se un tritessuto ¿ proiettivamente deformabile, in quanti modi, proiettivamente distinti, può essere deformato? L. Muracohin ha recentemente dimostrato in [16] che le trasformazioni puntuali fra due piani che sono proiettivamente deformabili dipendono da una funzione arbitraria di due argomenti. Segue facilmente da tale risultato che: se un tritessuto è proiettivamente deformabile, esso si può deformare, in generale, in un sol modo.

È già stato inoltre dimostrato da L. MuracchiNI [14], che un tritessuto può essere deformato al più in $\infty^{4}$ modi proiettivamente distinti. Si pone cusì il problema di determinare $\mathrm{j}$ tritessuti deformabili in $\infty^{h}(0 \leq h \leq 4)$ modi diversi e determinare anche le relative deformazioni proiettive. E questo è appunto lo scopo principale del presente lavoro, che o stato diviso in 5 paragrafi.

Nel § I viene introdotta una conveniente rappresentazione analitica del tritessuto, che lo definisce a meno di omografie, e vengono considerate alcune forme differenziali fratte invarianti, fra le quali un elemento lineare proiettivo che definisce il tritessuto a meno di deformazioni. Di tali forme differenziali si assegnano significati geometrici per mezzo di birapporti infinitesimi.

Nel \& II sono stabilite condizioni analitiche per la deformabilità di un tritessuto e si dimostra fra l'altro che, affinchè una corrispondenza fra due tritessati sia una deformazione proiettiva è necessario e sufficiente che questi abbiano lo stesso elemento lineare proiettivo.

Dopo aver ristabilito il risultato noto, secondo il quale un tritessuto è deformabile al più in $\infty^{4}$ modi, nel \& III, si determinano tutti i tritessuti che ammettono $\infty^{4}$ deformazioni. Si ottiene il risultato: $i$ tritessuti di curve piane che ammettono $\infty$ deformazioni proiettive sono tutti e soli quelli costituiti 
dalle tre famiglie $d i$ rette di un inviluppo algebrico di terza classe (anche riducibile). Le deformazioni proiettive di questi tritessuti sono già state studiate da O. BorÜWKa [5].

La ricerca dei tritessuti che posseggono $\infty^{3}$ deformazioni proiettive (\$ IV) conduce al risultato negativo: non esistono tritessuti di curve piane proiettivamente deformabili in $\infty^{3}$ modi diversi.

Infine nel $\S V$ vengono determinati $i$ tritessuti che posseggono $\infty^{2}$ deformazioni e si determinano anche le relative deformazioni. Una prima interessante proprietà di questi tritessuti, che semplifica notevolmente la ricerca, è che essi sono necessariamente a configurazione esagonale (a. 13). Una successiva proprietà di carattere analitico (nn. 14, 15, 16) apporta ulteriori semplificazioni, permettendo di superare le difficoltà algoritmiche che la ricerca presenta $e$ di giungere alla determinazione dei tritessuti richiesti. Alla loro effettiva determinazione si perviene considerando una notevole classe di superficie proiettivamente deformabili, già diffusamente studiate da B. SEGRE [17], le superficie $\Sigma$. Si tratta delle superficie possedenti una rete $R$ costituita dalle curve sezione con i piani passanti per una retta $r$ e dalle curve contorni apparenti dai punti di $r$. Proiettando da un punto $S$ di $r$ le asintotiche di $\Sigma$ e le curve sezione con i piani per $r$ si ottiene un tritessuto $\Delta_{\Sigma}$ che ammette $\infty^{2}$ deformazioni proiettive. Tali tritessuti, a meno di eventuali loro deformati proiettivi di tipo diverso, sono tutti e soli i tritessuti deformabili in $\infty^{2}$ modi. Si indica pure una costrazione delle $\infty^{2}$ deformazioni di tali tritessuti che conducono a tritessuti dello stesso tipo. Infine si determinano i tritessuti che ammettono due famiglie $\infty^{2}$ di deformazioni. Essi sono particolari tritessuti $\Delta_{\Sigma}$, provenienti da una ristretta famiglia di superficie $\Sigma$ rigate appartenenti ad una congruenza lineare.

Questi i problemi trattati e risolti nel presente lavoro. Molte altre questioni rimangono tuttavia aperte. Fra queste voglio ricordare le seguenti; a) studio dei tritessuti che ammettono deformazioni proiettive in sè; $b$ ) determinazione dei tritessuti che posseggono $\infty^{1}$ deformazioni ; c) determinazione (se esistono) dei tritessuti che ammettono un numero finito $>1$ di deformazioni proiettive.

Sul problema a) verrà apportato qualche contributo in un mio prossimo lavoro. Per quanto riguarda il problema b) si conosce una classe assai vasta di tritessuti deformabili in $\infty^{1}$ modi. Si tratta dei tritessuti formati da una generica rete asintotica e da un fascio di rette. Essi dipendono da una funzione arbitraria di due variabili. Ma purtroppo esistono altri tritessuti che ammettono $\propto^{1}$ deformazioni che non sono del tipo precedente nè loro deformati proiettivi $\left({ }^{8}\right)$.

(3) Tale è ad es. il tritessuto rappresentato dalle equazioni (3) e (4) dove i coefficienti $a, b, \beta, \gamma, \lambda$ sono tutte costanıi ed inoltre $\beta \cdot \gamma \cdot[3(b-a)+\beta-\gamma] \neq 0$. Le equazioni (12) am. mettono le $\infty^{t}$ soluzioni $X=$ cost. e non ne ammettono certo $\infty^{2}$ o piủ non essenco il t: $1-$ tessuto di nessuno del tipi determinati nel presenti lavoro. 


\section{$\S$ I - Forme invarianti ed elemento lineare proiettivo di un tritessuto.}

\section{Rappresentazione analitica di un tritessuto.}

Indicando con $x$ l'insieme delle tre coordinate proiettive omogenee di un punto di un piano $\pi$, consideriamo equazioni del tipo

$$
x=x(u, v),
$$

dove supporremo che le $x$ siano funzioni analitiche di due parametri $u$, $v$ e che il determinante $\left|x x_{u} x_{v}\right|$ () sia $\neq 0$. Fissate le (1), un tritessuto di curve di $\pi$ puo sempre rappresentarsi, limitatamente ad una regione sufficientemente piccola di $\pi$, con un'equazione del tipo

$$
A d u^{3}+B d u^{2} d v+C d u d v^{2}+D d v^{3}=0
$$

con $A, B, C, D$ funzioni analitiche di $u$ e $v$.

Assumendo i parametri $u \theta v$ in modo che due famiglie del tritessuto coincidano rispettivamente con le linee coordinate $u=$ cost. $e v=$ cost., la (2) diventa

$$
d u d v(d v-\lambda d u)=0
$$

dove $\lambda$ en funzione non nulla di $u$ e $v$.

Poichè moltiplicando le $x$ per un comune fattore $\rho(u, v) \neq 0$ il determinante $\left|x x_{u} x_{v}\right|$ viene ad essere moltiplicato per $p^{3}$, con scelta opportuna di $\rho$, si può sempre supporre che il determinante suddetto sia uguale ad una costante arbitraria non nulla. Scelto in tale guisa il fattore di proporzionalità delle coordinate, le funzioni $x$ risultano integrali di un sistema di equazioni differenziali, completamente integrabile, del tipo

$$
\begin{aligned}
& x_{u u}=-b x_{u}+\beta x_{v}+p x \\
& x_{u v}=a x_{u}+b x_{v}+c x \\
& x_{v v}=\gamma x_{u}-a x_{v}+q x,
\end{aligned}
$$

dove i coefficienti $a, b, \ldots$ sono funzioni di $u$ e $v$ soddisfacenti alle condizioni di completa integrabilità del sistema stesso.

Inversamente un sistema del tipo (4) completamente integrabile assieme alla (3) definisce, a meno di omografie, un tritessuto di curve piane. Poichè

(4) Qui e nel seguito indichiamo le derivazioni apponendo in basso le variabili rispetto a oui si deriva. 
nel seguito ci occuperemo di proprieta proiettive dei tritessuti, assumeremo come rappresentazione analitica del tritessuto le equazioni (3) e (4).

Le condizioni di integrabilità del sistema (4) si scrivono

$$
\begin{aligned}
& p=2 b^{2}+2 a \beta+b_{u}-\beta_{v} \\
& c=-a b+\beta \gamma-a_{u}-b_{v} \\
& q=2 a^{2}+2 b \gamma+a_{v}-\gamma_{u} \\
& a_{u u}+2 b_{u v}+3 b a_{u}+6 b b_{v}+3(a \beta)_{v}=\beta_{v v}+2 \gamma_{\gamma_{u}}+\gamma \beta_{u} \\
& b_{v v}+2 a_{u v}+3 a b_{v}+6 a a_{u}+3(b \gamma)_{u}=\gamma_{u u}+2 \gamma \beta_{v}+\beta \gamma_{v} .
\end{aligned}
$$

\section{Forme differenziali invarianti e loro significati.}

$\mathrm{E}$ ben noto come nella teoria delle reti piane si introducono delle forme differenziali fratte invarianti (le forme elementari e l'elemento lineare proiettivo) che giuocano un ruolo assai importante nella stessa teoria ( $\left.{ }^{5}\right)$.

Mediante le tre famiglie di curve di un tritessuto si possono costruire tre reti di curve piane e si possono quindi considerare anzitutto le due forme elementari relative a ciascuna rete. Si ottengono cosi sei forme differenziali fratte invarianti che, conservando la denominazione introdotta nella teoria delle superficie e delle reti piane, chiameremo le forme elementari del tritessuto e indicheremo con $f_{i j}\left({ }^{6}\right)$. Eseguendo i semplici calcoli si trovano per le $f_{i j}$ le espressioni :

$$
\begin{gathered}
f_{12}=\frac{\gamma d v^{2}}{d u}, \quad f_{21}=\frac{\beta d u^{2}}{d v}, \quad f_{31}=\frac{\eta d u^{2}}{d v-\lambda d u}, \\
f_{13}=\frac{\gamma(d v-\lambda d u)^{2}}{d u}, \quad f_{23}=\frac{\beta(d v-\lambda d u)^{2}}{\lambda^{2} d v}, \quad f_{32}=\frac{\eta d v^{2}}{\lambda^{2}(d v-\lambda d u)},
\end{gathered}
$$

dove

$$
\eta=3 \lambda(b-\lambda a)+\beta-\gamma \lambda^{3}+\lambda_{u}+\lambda \lambda_{v}
$$

Le forme (6) non sono indipendenti ma sono legate dalla relazione

$$
f_{12} f_{23} f_{31}=f_{18} f_{32} f_{21} \text {. }
$$

Osserviamo subito che l'annullarsi dei coefficienti $\gamma, \beta, \eta$ significa che sono rette le curve $u=$ cost.. $v=$ cost., $d v-\lambda d u=0$ rispettivamente.

(5) Si veda Fubini e С̆ech, [8], pp. 156-157.

(6) Un'interpretazione geometrica delle forme elementari è stata data dal CECH. Si veda: Fubini e delle superficie è dovuta al Bompianr [3]. 
Mediante le sei forme elementari si possono costruire i tre elementi lineari proiettivi delle reti formate dalle coppie di famiglie di curve del tritessuto. Essi sono le forme

$$
g_{i j}=\frac{1}{2}\left(f_{i j}+f_{j i}\right)
$$

Di essi è noto il seguente significato geometrico dovuto al CECH. Consideriamo una cubica $\Gamma_{i j}$ avente in un punto $x(u, v)$ un punto doppio a tangenti distinte e supponiamo che $\mathrm{i}$ due rami uscenti da $x$ abbiano ivi un contatto del $2^{\circ}$ ordine con le curve $C_{i}, C_{j}$ del tritessuto. Se $x^{\prime}(u+d u, v+d v)$ è un punto infinitamente vicino ad $x$ e $P_{i j}, Q_{i j}$ sono i punti d'intersezione della retta $x x^{\prime}$ con la cubica $\Gamma_{i j}$ e la sua retta dei flessi, si ha, a meno di infinitesimi d'ordine superiore,

$$
g_{i j}=-\left(x P_{i j} x^{\prime} Q_{i j}\right) \quad(i, j=1,2,3) .
$$

Le forme fratte precedenti sono già state considerate nella teoria delle reti piane, mentre non sono state considerate ancora le forme invarianti seguenti :

(8) $\quad \varphi_{1}=\frac{\gamma d v(\lambda d u-d v)}{d u}, \quad \varphi_{2}=\frac{\beta d u(d v-\lambda d u)}{\lambda d v}, \quad \varphi_{3}=\frac{\eta d u d v}{\lambda(\lambda d u-d v)}$.

Le forme $\varphi_{i}$ sono legate alle forme elementari dalle relazioni:

$$
\varphi_{1}^{2}=f_{12} f_{13}, \quad \varphi_{2}^{2}=f_{21} f_{23}, \quad \varphi_{3}^{2}=f_{31} f_{32} .
$$

Un'interpretazione geometrica delle $\varphi_{i}$, indipendente da quella nota delle $f_{i j}$, è la seguente :

si consideri una conica $\gamma_{i}$ avente in $x(u, v)$ un contatto del $2^{\circ}$ ordine con la curva $C_{i}$ del tritessuto $e$ sia $d_{i}$ la congingente $i$ punti intersezione di $\gamma_{i}$ con le tangenti in $x$ alle altre due curve del tritessuto. Se $x^{\prime} \dot{\partial}$ un punto infinitamente vicino ad $x$ e se $P_{i}, Q_{i}$ sono i punti intersezione della retta $x x^{\prime} \operatorname{con} \gamma_{i}$ e $d_{i}$ rispettivamente, a meno di infinitesimi d'ordine superiore, si ha:

$$
\varphi_{i}=2\left(x P_{i} x^{\prime} Q_{i}\right) \text {. }
$$

\section{Elemento lineare proiettiro.} fratta :

Chiameremo elemento lineare proiettivo del tritessuto la forma differenziale

(9) $\varphi=\varphi_{1}+\varphi_{2}+\varphi_{3}=\frac{\beta \lambda^{2} d u^{4}-2 \beta \lambda d u^{3} d v+\left(\beta-\eta-\gamma \lambda^{3}\right) d u^{2} d v^{2}+2 \gamma \lambda^{2} d u d v^{2}-\gamma \lambda d v^{4}}{\lambda d u d v(d v-\lambda d u)}$.

Allo scopo di assegnare un significato geometrico dell' elemento lineare proiettivo, vediamo dapprima come si possa associare intrinsecamente una 
retta covariante proiettiva ad una quartica piana con punto triplo a tangenti distinte.

Consideriamo il sistema lineare di quartiche individuato dalla quartica data e dalle tre quartiche riducibili formate con le tre coppie di tangenti nel punto triplo contate due volte. Esiste una ed una sola quartica del sistema che si spezza nelle tre tangenti suddette ed in una retta residua $r$.

La retta $r$ è una retta covariante proiettiva della quartica $(7)$.

Ciò premesso, si può assegnare il seguente significato geometrico dell'elemento lineare proiettivo:

consideriamo una quartica arente in $x(u, v)$ un punto triplo a tangenti distinte e supponiamo che $i$ tre rami uscenti da $x$ abbiano ivi un contatto del $2^{\circ}$ ordine con le tre curve del tritessuto. Se $x^{\prime}(u+d u, v+d v)$ è un punto infinitamente vicino ad $x$ e $P, Q$ sono i punti in cui la retta $x x^{\prime}$ incontra la quartica e la sua retta associata $r$, si ha, a meno di infinitesimi d'ordine superiore:

$$
\varphi=2\left(x P x^{\prime} Q\right) .
$$

Osserviamo anche che l' equazione ottenuta eguagliando a zero il denominatore di $\varphi$ rappresenta le direzioni delle tangenti in $x$ alle curve del tritessuto, mentre l'equazione ottenuta eguagliando a zero il numeratore rappre. senta le direzioni delle rette congiungenti $x$ con i punti d'intersezione di una qualunque delle quartiche sopradescritte con la relativa retta associata $r$.

\section{§ II - Deformazione proiettiva di un tritessuto.}

\section{Definizione di deformazione proiettiva.}

Una trasformazione $T$ fra due tritessuti $\Delta$ e $\Delta^{\prime}$ dicesi una deformazione proiettiva quando per ogni coppia $(x, y)$ di punti corrispondenti esiste un'omo. grafia che muta le curve di $\Delta$ passanti per $x$ in tre curve aventi in $y$ un contatto geometrico del $2^{\circ}$ ordine con le tre curve di $\Delta^{\prime}\left({ }^{8}\right)$.

Segue dalla definizione posta e da quella di curve caratteristiche di una trasformazione puntuale che, affinchè una trasformazione $T$ sia una defor-

(3) 11 procedimento qui usato per definire la retta $r$ è analogo ad altri indicati da $G$. Fupini nel trattato [7], rol. I, pp. 16.20, che generalizzano la retta dei flessi di una cubica con punto doppio a tangenti distinte e la retta principale di Tocliatri relativa ad una curva algebrica d'ordine $n$ con punto $(n-1)$-plo a tangenti distinte. Si veda a tale riguardo, oltre al trattato di Funin e CECH, la Nota [9] del Fubini.

(8) Questa definizicne è stata introdotta da L. Muracceini, [15]. Si osservi che se invece del contatto geometrico si esigesse il contatto analitico del $2^{\circ}$ ordine, le sole deformazioni proiettive sarebbero le omografie. 
mazione proiettiva di due tritessuti $\Delta, \Delta^{\prime}$ e necessario e sufficiente che $\Delta, \Delta^{\prime}$ siano $i$ due tritessuti caratteristici di $T$.

Si ha inoltre:

condizione necessaria e sufficiente affinch̀̀ una corrispondenza $T$ fra due tritessuti $\Delta, \Delta^{\prime}$ sia una deformazione proiettiva è che $i$ due tritessuti abbiano gli stessi elementi lineari proiettivi.

Supponiamo infatti che il tritessuto $\Delta$ sia quello rappresentato dalle equazioni (3) e (4). Per trasformare $\Delta$ in un nuovo tritessuto $\Delta^{\prime}$ basta assegnare delle nuove equazioni $y=y(u, v)$ o anche un nuovo sistema di equazioni differenziali analogo al sistema (4):

$$
\begin{aligned}
& y_{u u}=-\bar{b} y_{u}+\bar{\beta} y_{v}+\bar{p} y \\
& y_{u v}=\bar{a} y_{u}+\bar{b} y_{v}+\bar{c} y \\
& y_{v v}=\bar{\gamma} y_{u}-\bar{a} y_{v}+\bar{q} y,
\end{aligned}
$$

ed associare coppie di punti che si ottengono per gli stessi valori dei parametri $u$, v. Il tritessuto corrispondente $\Delta^{\prime}$ è quello rappresentato dalle equazioni (3) $\Theta\left(4^{\prime}\right)$. L'elemento lineare proiettivo di $\Delta^{\prime}$ è quello che si ottiene dalla (9) sostituendo $\beta, \gamma, \eta$ con $\bar{\beta}, \bar{\gamma}, \bar{\eta}(\bar{\eta}$ essendo data da formula analoga alla (7)).

Perchè gli elementi lineari proiettivi dei due tritessuti coincidano è necessario e sufficiente che si abbia $\beta=\bar{\beta}, \gamma=\bar{\gamma}, \eta=\bar{\eta}$. Ossia, sostituendo ad $\eta, \bar{\eta}$ i loro valori, deve aversi :

$$
\beta=\bar{\beta}, \quad \gamma=\bar{\gamma}, \quad \lambda a-b=\lambda \bar{a}-\bar{b} .
$$

D'altra parte affinchè la trasformazione $T$, rappresentata dalle $(4)$ \& $(4)$ sia una deformazione proiettiva di $\Delta, \Delta^{\prime}$ è necessario e sufficiente che le curve caratteristiche di $T$; rappresentate dall' equazione

$$
(\beta-\bar{\beta}) d u^{3}+3(b-\bar{b}) d u^{2} d v-3(a-\bar{a}) d u d v^{2}-(\gamma-\bar{\gamma}) d v^{3}=0,\left(^{9}\right)
$$

coincidano con le curve di $\Delta, \Delta^{\prime}$, ossia che l'equazione (10) coincida con la (3). Perchè ciò arvenga deve aversi :

$$
\beta=\bar{\beta}, \quad \gamma=\bar{\gamma}, \quad \lambda a-b=\lambda \bar{a}-\bar{b} .
$$

\section{Condizioni analitiche per la deformabilità di un tritessuto.}

Un tritessuto è in generale proiettivamente indeformabile mediante tra. sformazioni non omografiche $\left({ }^{10}\right)$. I tritessuti proiettivamente deformabili sono infatti $i$ tritessuti caratteristici delle trasformazioni puntuali e dipendono da

(9) Si reda ad es. Fubini e Čech. [8], pp. 160-161.

(19) Qui o nel seguito escluderemo sempre che la deformazione sia una omografia. 
due funzioni arbitrarie di due variabili, mentre un tritessuto generico dipende da tre funzioni di due variabili.

Pertanto se un tritessuto è proiettivamente deformabile le funzioni $\lambda$, $a, b, \ldots$ che compaiono nelle equazioni (3) e (4) dovranno soddisfare, oltre che alle condizioni di integrabilita (5), a nuove condizioni analitiche indipendenti dalle (5).

Assegnato un tritessuto di equazioni (3) e (4) perchè esso sia proiettivamente deformabile è necessario e sufficiente che esista una trasformazione puntuale per la quale il tritessuto è caratteristico.

In altri termini devono esistere delle equazioni $\left(4^{\prime}\right)$, in cui $\bar{\beta}=\beta, \bar{\gamma}=\gamma$, $\lambda \bar{a}-\bar{b}=\lambda a-b(\bar{a} \neq a$ e $\bar{b} \neq b)$, costituenti un sistema completamente inte. grabile. Ciò significa che devono esistere delle funzioni $\bar{a}$ e $\bar{b}$ f da $a$ e $b$ rispettivamente) soddisfacenti alle condizioni di integrabilità del sistema $\left(4^{\prime}\right)$ :

$$
\begin{aligned}
& \tilde{a}_{u u}+2 \bar{b}_{u v}+3 \bar{b} \bar{a}_{u}+6 \bar{b} \bar{b}_{v}+3(\bar{a} \beta)_{v}=\beta_{v v}+2 \beta \gamma_{u}+\gamma \beta_{u} \\
& \bar{b}_{v v}+2 \bar{a}_{u v}+3 \bar{a} \bar{b}_{v}+6 \bar{a} \bar{a}_{u}+3(\bar{b} \gamma)_{u}=\gamma_{u u}+2 \gamma \beta_{v}+\beta \gamma_{v} .
\end{aligned}
$$

Ponendo

$$
a-\bar{a}=X, \quad b-\bar{b}=\lambda X,
$$

e sottraendo membro a membro le ultime due equazioni (5) con le corrispondenti $\left(5^{\prime}\right)$, si hanno le equazioni

$$
\begin{array}{r}
X_{u u}+2 \lambda X_{u v}-3 \lambda X\left(X_{u}+2 \lambda X_{v}\right)-6 \lambda \lambda_{v} X^{2}+E X_{u}+F X_{v}+G X=0 \\
\lambda X_{v v}+2 X_{u v}-3 X\left(\lambda X_{v}+2 X_{u}\right)-3 \lambda_{v} X^{2}+L X_{u}+M X_{v}+N X=0
\end{array}
$$

dove

$$
\begin{array}{lll}
E=2 \lambda_{v}+3 b, & F=2 \lambda_{u}+6 \lambda b+33, & G=F_{v}+3 \lambda a_{u} \\
L=3 \lambda_{\gamma}+6 a, & M=2 \lambda_{v}+3 \lambda a, & N=L_{u}+\lambda_{v v}+3 \lambda_{v} a+3 b_{v} .
\end{array}
$$

Concludendo si ha dunque:

condizione necessaria e sufficiente affinchè il tritessuto di equazioni (3) $e$ (4) sia proiettivamente deformabile è che il sistema di equazioni differenziali (12) nella funzione incognita $X$ ammetta almeno una soluzione non nulla.

Osserviamo subito che se $X_{1}, X_{2}$ sono due soluzioni distinte e non nulle del sistema (12) esse individuano due trasformazioni puntuali $T_{1}, T_{2}$, omograficamente distinte che sono deformazioni proiettive del tritessuto di equazioni (3) $\mathrm{e}(4)$.

Ciò consegue dal fatto che se $X_{1} \neq X_{2}$ la trasformazione prodotto di $T_{1}$ per $T_{2}$ non è un' omografia poichè le sue direzioni caratteristiche non sono indeterminate $\left({ }^{11}\right)$.

(11) $\mathrm{E}$ ben noto che condizione necessaria e sufficiente affinchè una trasformazione puntuale sia un' omografia è che le direzioni caratteristiche siano indeterminate. 


\section{Tritessuti esagonali e tritessuti esagonali di rette.}

Allo scopo di procedere più speditamente nel seguito richiamiamo qui alcune nozioni di cui avremo frequentemente bisogno.

亡̀ dovata al THomsen ( ${ }^{(2)}$ la seguente nozione di tritessuto esagonale. Sia $x$ un punto generico e siano $C_{1}, C_{2}, C_{3}$ le carve della $1^{a}, 2^{a}, 3^{a}$ famiglia del tritessuto uscenti da $x$; sia $x_{1}$ un punto di $C_{1}$ prossimo ad $x$ e mandiamo per $x_{1}$ la curva della $3^{a}$ famiglia fino ad incontrare $C_{2}$ in un punto $x_{2}$; da $x_{2}$ mandiamo la curva della $1^{\text {a }}$ famiglia fino ad incontrare $C_{3}$ in $x_{3}$; e così di seguito fino ad ottenere su $C_{1}$ un punto $x_{7}$. Il tritessuto dicesi esagonale se, per ogni scelta di $x$ e $x_{1}$, si perviene ad un punto $x_{7}$ coincidente con $x_{1}$.

La condizione analitica che deve essere soddisfatta perchè il tritessuto di equazioni (3) e (4) sia esagonale si scrive

$$
(\log \lambda)_{u v}=0 .
$$

Se un tritessuto è esagonale $̀$ sempre possibile, con opportuna scelta dei parametri e del fattore di proporzionalità, fare in modo che si abbia

$$
\lambda=1 \text {. }
$$

Un esempio di tritessuto esagonale è costituito dalle rette di un inviluppo algebrico di terza classe (eventualmente riducibile) ed anzi, per un noto teorema di Graf e SAUER, ogni tritessuto esagonale di rette è formato dalle rette di un inviluppo algebrico di $3^{a}$ classe $\left({ }^{13}\right)$.

\section{§ III - Ricerca del tritessuti che ammettono $\infty^{4}$ deformazioni prolettive.}

\section{Prime conseguenze delle condizioni di deformabilità.}

Consideriamo le quattro equazioni differenziali del $3^{\circ}$ ordine ottenute per derivazione delle (12). Esse si scrivono:

$$
\begin{gathered}
X_{u u v}+2 \lambda X_{u u v}+(E-3 \lambda X) X_{u u}+\left(F+2 \lambda_{u}-6 \lambda^{2} X\right) X_{u v}-3 \lambda X_{u}{ }^{2}- \\
-6 \lambda^{2} X_{u} X_{v}-3\left(\lambda_{u}+4 \lambda \lambda_{v}\right) X X_{u}-12 \lambda \lambda_{u} X X_{v}+\left(E_{u}+G\right) X_{u}+F_{u} X_{v}- \\
-3\left(\lambda^{2}\right)_{u v} X^{2}+G u X=0 . \\
X_{u u v}+2 \lambda X_{u v v}+\left(E+2 \lambda_{v}-3 \lambda X\right) X_{u v}+\left(F-6 \lambda^{2} X\right) X_{v v}-3 \lambda X_{u} X_{v}-6\left(\lambda X_{v}\right)^{2}- \\
-3 \lambda_{v} X X_{u}-24 \lambda \lambda_{v} X X_{v}+E_{v} X_{u}+\left(F_{v}+G\right) X_{v}-3\left(\lambda^{2}\right)_{v v} X^{2}+G v X=0 .
\end{gathered}
$$

(12) Si veda: G. Thomsen, [18], p. 84.

(13) Si veda: H. GraF e R. SaUnR, [11]. Si veda anche: G. Fubini, [10]. 


$$
\begin{gathered}
2 X_{u u v}+\lambda X_{u v v}+(L-6 X) X_{u u}+(M-3 \lambda X) X_{u v}+\lambda_{u} X_{v v}-6 X_{u}{ }^{2}-3 \lambda X_{u} X_{v}- \\
-6 \lambda_{v} X X_{u}+3 \lambda_{u} X X_{v}+\left(L_{u}+N\right) X_{u}+M_{u} X_{v}-3 \lambda_{u v} X^{2}+N_{u} X=0 \\
2 X_{u v v}+\lambda X_{v v v}+(L-6 X) X_{u v}+\left(M+\lambda_{v}-3 \lambda X\right) X_{v v}-6 X_{u} X_{v}-3 \lambda X_{v}{ }^{2}- \\
-9 \lambda_{v} X X_{v}+L_{v} X_{u}+\left(M_{v}+N\right) X_{v}-3 \lambda_{v v} X^{2}+N_{v} X=0 .
\end{gathered}
$$

Da queste equazioni si possono dedurre univocamente le derivate terze della funzione incognita $X$, in funzione di $X$ e delle sue derivate prime e seconde, essendo nguale a $-3 \lambda^{2}$ il determinante dei coefficienti delle derivate terze. Ciò notoriamente ei assicura che il sistema (12) ammette al più infinite soluzioni dipendenti da quattro costanti arbitrarie. Segue dunque il risultato :

un tritessuto ammette al più $\infty^{4}$ deformazioni proiettive omograficamente distinte $\left({ }^{14}\right)$.

Come prima questione possiamo quindi proporci di ricercare i tritessuti che ammettono $\infty^{4}$ deformazioni proiettive.

\section{I tritessuti deformabili in $\infty^{4}$ modi.}

Perchè un tritessuto possegga $\infty^{4}$ deformazioni proiettive occorre che dal sistema (12) non si possano dedurre altre equazioni differenziali di ordine $\leq \dot{2}$ indipendenti dalle (12).

Consideriamo le quattro equazioni del $4^{\circ}$ ordine ottenute derivando le (17) e (18). In tali equazioni, che non scriveremo per brevità, compaiono soltanto tre derivate quarte di $X$ e cioe $X_{u u u v}, X_{u u v v}, X_{u v v v}$. Eliminando fra queste equazioni e le (12), (16), (17), (18), (19) le derivate quarte, terze e le derivate seconde $X_{u u}, X_{v v}$, si perviene ad un' equazione differenziale del tipo

$$
\begin{aligned}
R X_{u v}+S X_{u}^{2} & +2 T X_{u} X_{v}+U X^{2}{ }_{v}+\alpha X X_{u}+\delta X X_{v}+\varepsilon X_{u}+ \\
& +\vartheta X_{v}+\rho X^{3}+\sigma X^{2}+\tau X=0,
\end{aligned}
$$

dove i coefficienti sono funzioni di $u, v$ ed in particolare si ha:

$$
\begin{aligned}
& S=\frac{4 \eta}{\lambda}-\frac{4 \beta}{\lambda}-2 \lambda^{2} \gamma \\
& T=5 \eta+\beta-\lambda^{3} \gamma \\
& U=4 \lambda \eta+2 \lambda \beta+4 \lambda^{4} \gamma \\
& \rho=-\frac{9}{2} \lambda^{2}(\log \lambda)_{u v} .
\end{aligned}
$$

Derivando le (16) e (19) non si perviene a nuove equazioni di ordine $\leq \mathbf{2}$ in quanto due delle equazioni cosl ottenute coincidono con due già conside-

(14) Questo risultato era gia stato ottenuto da L. MuracchinI, [14] p. 421. 
rate sopra e due contengono le derivate quarte $X_{u u u u}, X_{v v v v}$ che non com* paiono nelle rimanenti equazioni.

Proviamo ora ohe:

$i$ tritessuti di curve piane che ammettono $\infty^{4}$ deformazioni proiettive sono tutti e soli quelli formati dalle rette di un inviluppo algebrico di $3^{a}$ classe (anche riducibile).

Infatti, per quanto si è detto sopra, perchè un tritessuto ammetta $\infty^{4}$ deformazioni proiettive è necessario che l'equazione differenziale (20) sia una identità e quindi, in particolare, che si abbia

$$
S=T=U=\rho=0 .
$$

Dalle (21), essendo $\lambda \neq 0$, seguono allora le relazioni

$$
\beta=\gamma=\eta=0, \quad(\log \lambda)_{\mu v}=0 .
$$

Le prime tre ci assicurano che il tritessato è formato di rette e l'ultima (si veda n. 6) che il tritessuto $\dot{e}$ a configurazione esagonale Per il teorema di Graf e SAUER il tritessuto è quindi formato dalle rette di un inviluppo algebrico di terza classe.

E noto viceversa che $\mathrm{i}$ tritessuti costituiti dalle rette di un inviluppo algebrico di $3^{\text {a }}$ classe ammettono $\infty^{*}$ deformazioni proiettive $\left({ }^{25}\right)$.

\section{§ IV - Ricerca dei tritessuti che ammettono $\infty^{3}$ deformazioni prolettive.}

\section{Conseguenze differenziali della (20).}

Ci possiamo ora chiedere se esistano dei tritessuti che ammettono $\infty^{3}$ deformazioni proiettive. Proveremo in questo paragrafo che non esistono tritessuti siffatti.

Perchè un tritessuto possegga $\infty^{3}$ deformazioni è necessario e sufficiente che le equazioni differenziali (12) $\Theta$ (20) costituiscano un sistema chinso, ossia non ammettano altre conseguenze differenziali indipendenti di ordine $\leq 2$. Per rispondere al quesito propostoci occorrerà quindi analizzare le conseguenze differenziali della (20).

Derivando la (20) ed eliminando dalle equazioni ottenute le derivate terze mediante le (17) e (18) e le derivate seconde $X_{u u}, X_{v v}$ mediante le (12), si

(15) Si veda O. BorüWKa, [5]. Alla pag. 22 di questo lavoro è assegnata anche una costruzione delle deformazioni proiettive di un tritessuto esagonale di rette. 
perviene alle due equazioni :

$$
\begin{gathered}
\left(A_{1} X_{u}+A_{2} X_{v}+A_{3} X+A_{4}\right) X_{u v}=A_{5} X X_{u}{ }^{2}+A_{6} X X_{u} X_{v}+A_{7} X X_{v}{ }^{2}+A_{8} X^{2} X_{u}+ \\
+A_{9} X^{2} X_{v}+A_{10} X^{s}+P_{2}\left(X, X_{u}, X_{v}\right) \\
\left(B_{1} X_{u}+B_{2} X_{v}+B_{3} X+B_{4}\right) X_{u v}=B_{8} X X_{u}{ }^{2}+\ldots+B_{10} X^{3}+Q_{2}\left(X, X_{u}, X_{v}\right)
\end{gathered}
$$

dove si ha in particolare:

$$
\begin{aligned}
& A_{1}=\lambda S, \quad A_{2}=\lambda T, \quad A_{5}=2 \lambda S+2 T, A_{6}=4 \lambda^{2} S+3 \lambda T+2 U ., \\
& A_{7}=4 \lambda^{2} T+\lambda U, \\
& B_{1}=T, \quad B_{2}=U, \quad B_{5}=\lambda S+4 T, \quad B_{6}=2 \lambda^{2} S+3 \lambda T+4 U, \\
& B_{7}=2 \lambda^{2} T+2 \lambda U,
\end{aligned}
$$

e $P_{2}, Q_{2}$ sono polinomi di $2^{\circ}$ grado in $X, X_{u}, X_{v}$.

Conviene ora distinguere due casi secondochè $R=0$ oppure $R \neq 0$.

10. Caso $R=0$.

Se $R=0$ l'equazione differenziale $(20)$ è del $1^{\circ}$ ordine e quindi perchè il sistema costituito dalle (12) e (20) sia chiuso occorre che le (22) e (23) siano loro conseguenze. Perchè ciò avvenga nelle (22) e (23) non dovrà in partico. lare, comparire la derivata seconda $X_{u v}$. Dovrà quindi aversi :

$$
\begin{aligned}
& S X_{u}+T X_{v}+H X+K=0 \\
& T X_{u}+U X_{v}+H^{\prime} X+K^{\prime}=0,
\end{aligned}
$$

dove $H, K, H^{\prime}, K^{\prime}$ sono funzioni il cui valore non interessa nel seguito. Le (24) devono essere identita o conseguenze della (20). Nel primo caso si ha $S=T=U=0$. Nel secondo caso derivando le (24) rispetto ad $u$ e $v$ ed eliminando le derivate seconde $X_{u u}, X_{v n}$, si ottengono le quattro equazioni

$$
\begin{array}{ll}
(T-2 S) X_{u v}+\ldots=0, & (S-2 T) X_{u v}+\ldots=0, \\
(U-2 T) X_{u v}+\ldots=0, & (T-2 U) X_{u v}+\ldots=0,
\end{array}
$$

dove si sono omessi $i$ termini contenati le derivate prime e la funzione. In queste equazioni devono essere nulli $i$ ooefficienti di $X_{u v}$, onde si ha ancora $S=T=U=0$.

Avendo riguardo ai valori di $S, T, U$ dati dalle (21), segue

$$
\beta=\gamma=\eta=0 \text {. }
$$

Le (25) ci assicurano che il tritessuto è costituito da tre famiglie di rette (vedasi il n. 2). Ora se il tritessuto di rette d̀ a configurazione esagonaie possiede $\infty^{4}$ e non $\infty^{3}$ deformazioni, mentre, se non è.tale, per un teorema 
dornto a G. BoL $\left({ }^{16}\right)$, possiede al più 17 deformazioni proiettive. Si conclude dunque :

nel caso $R=0$ il tritessuto non possiede mai $\infty^{3}$ deformazioni proiettive (esattamente).

\section{Caso $R \neq 0$.}

Nel caso in cui $R \neq 0$, eliminando nelle (22), (23) la derivata $X_{u v}$ mediante la (20), si ottengono due equazioni differenziali del $1^{\circ}$ ordine. Perchè il sistema (12), (20) sia chiuso occorre che tali equazioni siano identicamente soddisfatte. Uguagliaudo a zero $\mathrm{i}$ coefficienti di $X_{u}{ }^{3}, X_{u}{ }^{2} X_{v}, X_{u} X_{v}{ }^{2}, X_{v}{ }^{3}$, si ha manifestamente $S=T=U=0$.

Anche nel caso $R \neq 0$ si perviene dunque alla stessa conclusione negativa a cui si $\dot{\theta}$ giunti per il easo $R=0$. Concludendo:

non esistono tritessuti di curve piane che ammettono $\infty^{3}$ deformazioni proiettive.

\section{$\S \nabla$ - Ricerca dei tritessuti che ammettono $\infty^{2}$ deformazioni prolettive.}

\section{Una elasse di tritessuti che posseggono $\infty^{2}$ deformazioni.}

Ci si può ora chiedere se esistano dei tritessuti che posseggono $\infty^{2}$ deformazioni proiettive e, in caso affermativo, quali siano tali tritessuti e quali le loro deformazioni.

In questo n. mostreremo innanzitutto che tritessuti siffatti esistono, segnalando una certa classe di tritessuti che godono della proprieta voluta.

B. SegRe $\left({ }^{17}\right)$ in una interessante Memoria del 1931, dedicata alla teoria dell' applicabilità proiettiva della saperficie, ha diffusamente studiato, assieme ad altre, una classe di superficie proiettivamente deformabili, chiamate superficie $\Sigma$, che sono caratterizzate dalla proprieta di possedere una rete $R$ costituita dalle curve sezione della superficie con $i$ piani passanti per una retta $r$ e dalle curve contorni apparenti dai panti di $r$. Ora si ha che:

fissata nello spazio ordinario una superficie $\Sigma$ e detta $r$ l'asse del fascio di piani contenenti una famiglia di curve della rele $R$ di $\Sigma$, proiettando da un punto. $S$ di $r$ su un piano $\pi$ le asintotiche di $\Sigma$ e le curve sezione con $i$ piani per $r$, si ottiene un tritessuto di curve piane deformabile in $\infty^{2}$ modi.

(16) Si veda: G. BoL [2]* p. 45. Ricordo che secondo un'ipotesi di H. F. Gronwall, noia col nome di teorema ai unicita della nomografia, i soli tritessuti di rette proiettivamente deformabili sono quelli a configurazione esagonale (si veda: H. F. Growwall, [12]). Di tale ipotesi non si possiede ancora nè una dimostrazione nè una smentita.

(17) Si reda: B. SEGRE, [17]. 
Infatti ogni superficie $\Sigma$ ammette $\infty^{1}$ deformazioni proiettive che la trasformano ancora in una superficie $\Sigma$ e mntano le curve giacenti sui piani per $r$ in curve appartenenti ai piani di un fascio avente per asse una certa retta $r^{\prime}\left({ }^{18}\right)$.

Si consideri ora la trasformazione $T$ fra due piani $\pi, \pi^{\prime}$ ottenuta associando coppie di punti $\left(P, P^{\prime}\right)$ che sono proiezioni rispettivamente da un punto $S$ di $r$ e da un punto $S^{\prime}$ di $r^{\prime}$ di coppie di punti corrispondenti delle due superficie. La trasformazione $T$ ha come curve caratteristiche su $\pi$ lo proiezioni da $S$ delle asintotiche di $\Sigma$ e delle curve sezione con i piani per $r$. Invero la deformazione fra le due superficie conserva i flessi delle curve tangenti alle curve asintotiche e quindi le proiezioni delle asintotiche su $\pi$ godono della stessá proprietà e sono curve caratteristiche per ' 'í. Infine le proiezioni da $S, S^{\prime}$ delle curve sezione delle due superficie con i piani per $r, r^{\prime}$ sono rette corrispondenti e perciò curve caratteristiche di $T$.

Indichiamo col simbolo $\Delta_{\Sigma}$ i tritessuti ottenuti con la costruzione prece. dente. Si ha:

$i$ tritessuti $\Delta_{\Sigma}$ posseggono $\infty^{2}$ deformazioni proiettive che si ottengono considerando una qualunque deformazione della superficie $\Sigma$ che muti le curve piane della rete $R$ in curve piane, ed associando le proiezioni di punti corrispondenti delle due superficie da due punti $S, S^{\prime}$ appartcnenti agli assi dei fasci di piani contenenti le curve della rete $R$.

L'interesse di questi tritessuti risiede soprattutto nel fatto che, come verrà provato nei nn. seguenti, essi, assieme a loro eventuali deformati pro* iettivi di tipo diverso, sono gli unici tritessuti che ammettono $\infty^{2}$ deformazioni.

\section{Una prima proprietà.}

Perchè un tritessuto fossegga $\propto^{2}$ deformazioni è necessario e sufficiente che soltanto quattro delle cinque equazioni differenziali (12), (20), (22), (23) siano indipendenti ed inoltre che queste quattro equazioni costituiscano un sistema chiuso. Per la effettiva determinazione di tali tritessuti bisogna quindi tener conto di entrambe queste condizioni. Ora, non appena si rifletta sugli sviluppi analitici cui esse danno luogo, vien fatto di pensare che la ricerca si debba fatalmente arenare nella complicazione algoritmica. Invero i calcoli che si presentano, se affrontati in modo pedante, non portano a nessuna conclusione.

Le cose vengono invece assai semplificate da una prima notevole proprietà geometrica. Dimostreremo che:

(18) ì noto the ogni superficie che possiede una rete $R$ ammette esaltamente $\infty^{1}$ deformazioni proiettive per le quali la rete $\boldsymbol{h}$ è rete di deformazione. 
I tritessuti che posseggono $\infty^{2}$ deformazioni proiettive sono necessariamente a configurazione esagonale.

Questa proprietà, oltre che per sè stessa interessante, permette una semplificazione assai notevole nei calcoli, poichè, per i tritessuti esagonali, ossia quelli per cui $\rho=0$, si possono sempre scegliere $i$ parametri in modo che sia $\lambda=1$ (si veda il n. 6 ).

Dimostriamo dunque dapprima il teorema enunciato, distinguendo $\mathrm{i}$ due casi : a) $R=0$, b) $R \neq 0$.

a) Se $R=0$, i coefficienti di $X_{u v}$ che compaiono nelle (22), (23) non possono essere tutti e due identicamente nulli, poichè ne seguirebbe $S=T=$ $=U=0$ e quindi, per quanto è stato ricordato al n. 10 il tritessuto sarebbe formato da rette e non potrebbe possedere $\infty^{2}$ deformazioni. Nè può darsi che tali coefficienti siano nulli in conseguenza della (20). In tal caso infatti il polinomio in $X, X_{u}, X_{v}$, che compare al $1^{\circ}$ membro della (20) dovrebbe essere riducibile. Ma ciò, se $\rho \neq 0$ (cioè il tritessutọ non è esagonale), condurebbe alle condizioni $S=T=U=0$.

Il sistema di equazioni (12), (20), (22), (23), se $\rho \neq 0$, si può cosi trasformare in altro equivalente costituito da tre equazioni del $2^{\circ}$ ordine dalle quali possono dedursi le derivate $X_{u u}, X_{u v}, X_{v v}$ in funzione delle derivate prime e della funzione incognita $X$, e da due equazioni del $1^{\circ}$ ordine: la (20) e quella ottenuta eliminando $X_{u v}$ dalle (22), (23). del tipo

L'equazione del $1^{\circ}$ ordine ottenuta dalle (22), (23) eliminando $X_{u v}$ è

$$
\begin{gathered}
X\left(C_{1} X_{u}{ }^{3}+C_{2} X_{u}{ }^{2} X_{v}+C_{3} X_{u} X_{v}{ }^{2}+C_{4} X_{v}{ }^{3}\right)+X^{2} H_{2}\left(X, X_{u}, X_{v}\right)+ \\
X^{3} H_{1}\left(X, X_{u}, X_{v}\right)+H_{3}\left(X, X_{u}, X_{v}\right)=0,
\end{gathered}
$$

dove $H_{1}, H_{2}, H_{3}$ sono polinomi in $X, X_{u}, X_{v}$ di grado uguale all'indice ed inoltre si ha

$$
\begin{array}{ll}
C_{1}=2 T^{2}-\lambda S(\lambda S+2 T), & C_{2}=2 \lambda^{3} S^{2}-2 \lambda S U-\lambda T^{2}+4 U T, \\
C_{8}=2 U^{2}+2 \lambda^{2} U S+\lambda^{2} T^{2}-4 \lambda^{3} S T, & C_{4}=-2 \lambda^{3} T^{2}+U\left(\lambda U+2 \lambda^{2} T\right) .
\end{array}
$$

Le equazioni $(20)$ e (26) debbono essere l'una consegnenza dell'altra. Consideriamo lo spazio a tre dimensioni di coordinate non omogenee $X, X_{u}$, $X_{v}$. In tale spazio le equazioni (20) (nella quale $R=0$ ) e la (26) rappresentano rispettivamente una superficie cubica e una superficie del $4^{\circ}$ ordine. Si vede subito che la superficie cubica di equazione (20) è irriducibile, nell'ipotesi $\rho \neq 0$, non potendo sussistere le tre condizioni $S=T=U=0$. Infatti siccome incontra il piano improprio secondo la retta all'infinito del piano $X=0$ contata tre volte, per essere riducibile dovrebbe contenere un piano di equazione $X=K$ il che conduce necessariamente alle condizioni $S=T=U=0$.

Essendo tale superficie cubica irriducibile, dovrà far parte della.superficie 
del $4^{\circ}$ ordine di equazione (26). In particolare la curva intersezione della superficie (26) col piano improprio dovrà contenere la retta impropria del piano $X=0$, onde

$$
C_{1}=C_{2}=C_{3}=C_{4}=0 .
$$

Dalle (27) seguono le tre condizioni impossibili $S=T=U=0$.

b) $S_{e} R \neq 0$, eliminando $X_{u v}$ dalle (22), (23), per mezzo della (20), si ottengono due equazioni differenziali del $1^{\circ}$ ordine del tipo:

$$
\begin{gathered}
\frac{1}{R}\left(A_{1} X_{u}+A_{2} X_{v}+A_{3} X+A_{4}\right)\left(\rho X^{3}+S X_{u}{ }^{2}+2 T X_{u} X_{v}+U X_{v}{ }^{2}+\right. \\
\left.+\alpha X X_{u}+\delta X X_{v}+\sigma X^{2}\right)+X\left(A_{5} X_{u}{ }^{2}+A_{6} X_{u} X_{v}+A_{7} X_{v}{ }^{2}\right)+X^{2}\left(A_{8} X_{u}+\right. \\
\left.+A_{9} X_{v}\right)+A_{10} X^{3}+K_{2}\left(X, X_{u}, X_{v}\right)=0 \\
\frac{1}{R}\left(B_{1} X_{u}+B_{2} X_{v}+B_{s} X+B_{4}\right)\left(\rho X^{3}+S X_{u}{ }^{2}+2 T X_{u} X_{v}+U \nabla_{v}{ }^{2}+\right. \\
\left.+\alpha X X_{u}+\delta X X_{v}+\sigma X^{2}\right)+X\left(B_{5} X_{u}{ }^{2}+B_{8} X_{u} X_{v}+B_{7} X_{v}{ }^{2}\right)+\ldots+ \\
+B_{10} X^{3}+K_{2}^{\prime}\left(X, X_{u}, X_{v}\right)=0
\end{gathered}
$$

dove $\rho, S, T, U, \alpha, \delta, \sigma$ sono gli stessi coefficienti che compaiono nella (20) e le $A_{i}$ e $B_{i}$ sono i coefficienti che compaiono nelle (22) e (23) ed infine $K_{2}$ e $K_{2}^{\prime}$ sono polinomi di $2^{\circ}$ grado in $X, X_{u}, X_{v}$.

Supposto $\rho \neq 0$, distinguiamo due sottocasi: 1) le due superficie dell' $S_{3}$ $\left(X, X_{u}, X_{v}\right)$ di equazioni (28) e (29) sono irriducibili; 2) le dae superficie sono ridnoibili.

Nel caso 1) le due equazioni $(28) \Theta$ (29) devono coincidere. Ton potendo essere $S=T=U=0$, supponiamo ad es. $S \neq 0$. Per la coincidenza dovrà essere, in particolare,

$$
T=\mu S, \quad U=\mu^{2} S, B_{s}=\mu A_{s} \quad(\mu \neq 0) .
$$

Moltiplichiamo i primi membri della (28), (29) rispettivamente per $\mu$ e $\lambda$. Per la coincidenza delle due equazioni è ora necessario che i primi membri siano polinomi identici e quindi si avrà, in particolare,

$$
\mu A_{5}=\lambda B_{5} \quad, \quad \mu A_{8}=\lambda B_{6} \quad, \mu A_{7}=\lambda B_{7} .
$$

Le (30) coesistono solo se $S=0$ contro l'ipotesi.

Nel caso 2) supponiamo sempre $S \neq 0$. Si vede allora che la superficie (28) si può spezzare eventualmenle in un unico modo e cioè in un piano $\theta$ una superficie cubica. Infatti nel caso opposto la retta all'infinito del piano $X=0$ dovrebbe essere comune a due componenti della superficie (essendo retta tripla della curva intersezione della superficie col piano improprio) e 
quindi luogo di punti multipli per la snperficie di equazione (28). Ma ciò non è possibile se $S \neq 0$.

Si può ancora osservare che le due superficie non possono avere un piano comune. In tal caso la $X$ sarebbe integrale di un'equazione differenziale del $1^{\circ}$ ordine del tipo

$$
A X_{u}+B X_{v}+C X+D=0 \text {. }
$$

Orbene derivando tale equazione si ottengono due nuove equazioni che, assieme alle (12), permettono di ottenere le derivate seconde in funzione di ura delle derivate prime e della $X$. Perchè con questa coesista la (20) deve essere $p=0$, contro l'ipotesi.

Perchè le due equazioni (28) e (29) siano l'una conseguenza dell'altra, rimane come unica possibilità solo il caso in cui le due superficie abbiano in comune una superficie cubica. Si vede allora che tale superficie deve avere necessariamente equazione del tipo

$$
\begin{gathered}
S X_{u}^{2}+2 T X_{u} X_{v}+U X_{v}^{2}+p X^{3}+\bar{x} X X_{u}+\bar{\delta} X X_{v}+\bar{z} X^{2}+ \\
+P_{1}\left(X, X_{u}, X_{v}\right)=0
\end{gathered}
$$

dove $S, T, U, \rho$ sono gli stessi coefficienti che compaiono nella (20) e $P_{1}$, ⿺̀ un polinomio di $1^{\circ}$ grado in $X, X_{w}, X_{v}$.

Ma procedendo ora per la (31) nello stesso modo gia usato per la (20) nel caso $R=0$, si prova che non può essere $p \neq 0$. Invero gli unici coefficienti di cui si è tenuto conto in quei ragionamenti sono $S, T, U$, che sono uguali per le due equazioni (20) e (31).

Il teorema enunciato in principio è così dimostrato.

\section{Una seconda proprietà.}

Per la proprietà dimostrata nel $n$. precedente, possiamo ora limitarci a considerare $i$ tritessuti a configarazione esagonale, ossia quelli per cui

$$
\lambda=1 \text {. }
$$

Le equazioni (12), (20), (22), (23), nel caso $\lambda=1$ sono notevolmente più semplici e maneggevoli. In particolare, per i coefficienti $R, S, T, U, \alpha, \delta$, che appaiono nella (20) si ha

$$
\begin{aligned}
& R=2\left(\eta_{u}+\eta_{v}+\beta_{u}-\gamma_{v}\right) \\
& S=4 \eta-4 \beta-2 \gamma \\
& T=5 \eta+\beta-\gamma \\
& U=4 \eta+2 \beta+4 \gamma \\
& \alpha=-6\left(\eta_{u}+\beta_{u}-\beta_{v}+2 \gamma_{u}\right) \\
& \delta=-6\left(\eta_{v}-2 \beta_{v}+\gamma_{u}-\gamma_{v}\right)
\end{aligned}
$$


mentre per i coefficienti delle (22), (23), (28), (29) si ha

$$
\begin{aligned}
& A_{1}=S, \quad A_{2}=T, \quad A_{3}=\frac{1}{2}(x+3 R), \\
& A_{5}=2(S+T), \quad A_{0}=4 S+3 T+2 U, \quad A_{7}=4 T+U, \\
& A_{8}=\alpha+\delta+3 R, \quad A_{9}=2 \alpha+\frac{\delta}{2}+6 R . \\
& B_{1}=T, \quad B_{2}=U, \quad B_{3}=\frac{1}{2}(\delta+3 R), \\
& B_{5}=S+4 T, \quad B_{6}=2 S+3 T+4 U, \quad B_{7}=2(T+U), \\
& B_{8}=\frac{\alpha}{2}+2 \delta+6 R, \quad B_{9}=\alpha+\delta+3 R .
\end{aligned}
$$

Nei due numeri successivi dimostreremo la seguente proposizione:

Affinchè un tritessuto possegga $\infty$ 'deformazioni proiettive è necessario che dalle equazioni (12), (20), (22), (23) si possa dedurre una equazione del $1^{\circ}$ ordine lineare in $X, X_{w}, X_{v}$ compatibile con le (12).

In altri termini il sistema (12) deve essere prolungabile soltanto mediante un' equazione del $2^{\circ}$ ordine e una del $1^{\circ}$, ed inoltre questa equazione del $1^{\circ}$ ordine deve essere necessariamente lineare.

Per la dimostrazione è ancora opportuno distinguere i due casi : a) $R=0$, b) $R \neq 0$.

15. a) Caso $R=0$.

Se $R=0$, l' equazione differenziale del $1^{\circ}$ ordine che si ottiene eliminando $X_{u v}$ dalle (22), (23) ¿े del tipo

$$
\begin{gathered}
X\left(C_{1} X_{u}{ }^{3}+C_{2} X_{u}{ }^{2} X_{v}+C_{3} X_{u} X_{v}{ }^{2}+C_{4} X_{v}{ }^{3}\right)+X^{2}\left(C_{5} X_{u}{ }^{2}+C_{8} X_{u} X_{v}+C_{7} X_{v}{ }^{2}\right)+ \\
+X\left(C_{8} X_{u}+C_{8} X_{v}\right)+H_{3}\left(X, X_{u}, X_{v}\right)=0
\end{gathered}
$$

dove $H_{8}$ è un polinomio di $3^{\circ}$ grado in $X, X_{u}, X_{v}$ ed inoltre

$$
\begin{aligned}
& C_{2}=S^{2}+2 S T-2 T^{2}, \quad C_{2}=2 S^{2}+2 S U+T^{2}-4 U T, \\
& C_{3}=4 S T-T^{2}-2 S U-2 U^{2}, \quad C_{4}=2 T^{2}-2 U T-U^{2} \text {. } \\
& C_{5}=\alpha S+\alpha T+\delta S-2 \delta T, \quad C_{6}=2 \alpha S+\alpha U-\delta S-2 \delta U, \\
& C_{7}=2 \alpha T-\alpha U-\delta T-\delta U, \quad 4 C_{8}=\alpha^{2}+2 \alpha \delta-2 \delta^{2} \text {, } \\
& 4 C_{9}=2 \alpha^{2}-2 \alpha \delta-\delta^{2} .
\end{aligned}
$$

Supponiamo per assurdo che non sia vero il teorema. Allora, poichè non -può sussistere più di un'equazione del $1^{\circ}$ ordine, il primo membro della (20) deve essere un polinomio irriducibile e per esso deve essere divisibile il primo membro della (35). 
Limitandoci a considerare i termini di grado più elevato dei due polinomi, per la divisibilità dovranno esistere tre funzioni $J, H, K$ per cui

$$
\begin{gathered}
C_{1}=J S, \quad C_{2}=H S+2 J T, \quad C_{3}=2 H T+J U, \quad C_{4}=H U \\
C_{5}=K S+J \propto, \quad C_{6}=2 K T+H \alpha+J \delta, \quad C_{7}=K U+H \delta, \\
C_{8}=K \alpha+J \sigma, \quad C_{9}=K \delta+H \sigma, \quad K \sigma=0 .
\end{gathered}
$$

Eliminando $H$ e $J$ dalle prime quattro delle (37), si hanno le due condizioni

$$
\begin{aligned}
& \left(U S-T^{2}\right)(5 S-4 T)(S+U+2 T)=0, \\
& \left(U S-T^{2}\right)(S-2 T)(S-4 T+4 U)=0 .
\end{aligned}
$$

Conviene ora distinguere due sottocasi: 1) $U S-T^{2} \neq 0$; 2) $U S-T^{2}=0$.

Nel sottocaso 1), essendo $U S-T^{2} \neq 0$, dalle (38) si deduce che devono necessariamente sussistere due delle tre condizioni $\beta=0, \gamma=0, \eta=0$, che assicurano essere rette rispettivamente le curve del tritessuto $v=$ cost., $u=$ cost., $u-v=$ cost.

Supponiamo ad es. $\beta=\gamma=0, \eta \neq 0\left({ }^{19}\right)$ e ciò̀ $U=S, 4 T=5 S, S \neq 0$. Dalle (37) si ha allora:

$$
8 K=15 \alpha-12 \delta, \quad 20 K=21 x-21 \delta, \quad 8 K=12 \alpha-15 \delta,
$$

dalle quali segue

$$
\alpha=\delta=K=0 .
$$

Per le (33) si ha $\eta_{u}=\eta_{v}=0$, ossia

$$
b-a=k,
$$

dove $k$ è una costante non nulla, essendo $\eta \neq 0$.

Le condizioni di integrabilità (5) del sistema di equazioni differenziali (4) che rappresenta il tritessuto nel caso attuale si scrivono

$$
\begin{aligned}
& a_{u u}+2 a_{u v}+3 a\left(a_{u}+2 a_{v}\right)+3 k\left(a_{u}+2 a_{v}\right)=0, \\
& a_{v v}+2 a_{u v}+3 a\left(2 a_{u}+a_{v}\right)=0 .
\end{aligned}
$$

Queste equazioni coincidono con quelle ottenute dalla (12) ove si pongano $X=-a, \quad \lambda=1, \quad E=-3 k, \quad F=-6 k, \quad G=L=M=N=0$. Perchè siano compatibili deve aversi perciò l' equazione analoga alla (20) che si scrive

$$
\left(a_{u}+2 a_{v}\right)\left(2 a_{u}+a_{v}\right)=0 .
$$

(\$9) Alle stesse conclusioni si arriva ovviamente negli altri due casi possibili. Non può supporsi $\beta=\gamma=\eta=0$ per quanto è stato osservato al n. 10 . 
Ma allora si vede subito che il sistema (12) è compatibile se viene prolungato con una delle due equazioni lineari.

$$
X_{u}+2 X_{v}=0, \quad 2 X_{u}+X_{v}=0
$$

contro l' ipotesi.

Nel sottocaso 2), essendo $U S-T^{2}=0$, non può aversi $S=U=0$ poichè seguirebbe anche $T=0$. Supponiamo dunque ad es. $S \neq 0$. Si avrà allora

$$
\begin{aligned}
& T=\mu S, \quad U=\mu^{2} S, \\
& J=\left(1+2 \mu+2 \mu^{2}\right) S, \quad H=\left(2-2 \mu-\mu^{2}\right) S, \quad K=(1-2 \mu)(\delta-\alpha \mu) .
\end{aligned}
$$

Sostituendo nella (37) i valori dati dalle (39) si hanno in particolare le due relazioni

$$
(\overline{0}-\alpha \mu)\left(2-\mu+\mu^{2}-2 \mu^{3}\right)=0, \quad(\delta-\alpha \mu)\left(1+2 \mu-2 \mu^{2}\right)=0
$$

dalle quali segue necessariamente

$$
\delta=\alpha \mu . \quad K=0 .
$$

Dalle ultime equazioni (37) si ha inoltre

$$
\left(\alpha^{2}-4 \sigma S\right)\left(1+2 \mu-2 \mu^{2}\right)=0, \quad\left(\alpha^{2}-4 \sigma S\right)\left(2-2 \mu-\mu^{2}\right)=0,
$$

da cui si ha

$$
\alpha^{2}-4 \sigma S=0 .
$$

Per le (39), (40) e (41), la (20) è necessariamente del tipo

$$
\left(X_{u}+\mu X_{v}+\nu X\right)^{2}+2 \varepsilon X_{u}+2 \mathcal{v} X_{v}+2 \tau X=0 .
$$

L' equazione del $1^{\circ}$ ordine ottenuta derivando la (42) ed eliminando le derivate seconde, avendo riguardo alla (42) stessa, si scrive

$$
X\left(D_{1} X_{u}{ }^{2}+D_{2} X_{u} X_{v}+D_{3} X_{v}{ }^{2}+D_{4} X X_{u}+D_{5} X X_{v}\right)+H_{2}\left(X, X_{u}, X_{v}\right)=0
$$

dove $H_{2}$ è un polinomio di $2^{\circ}$ grado in $X, X_{\mu}, X_{v}$ ed inoltre

$$
\begin{aligned}
& D_{1}=(\vartheta-\mu \varepsilon)(1-2 \mu), \quad D_{2}=-2(\vartheta-\mu \varepsilon)(1+\mu), \quad D_{3}=(\vartheta-\mu \varepsilon)(-2+\mu), \\
& D_{4}=\tau\left(-1-2 \mu+2 \mu^{2}\right)+v\left(\varepsilon+\varepsilon \mu+\vartheta-2 \vartheta(\eta), \quad D_{5}=\tau\left(-2+2 \mu+\mu^{2}\right)+v(2 \varepsilon-\varepsilon \mu-\vartheta-\vartheta \mu) .\right.
\end{aligned}
$$

Perchè la (43) sia conseguenza della (42) dovranno necessariamente verificarsi una delle due eventualità : o nelle (43) sono nulli tutti i coefficienti dei termini di $3^{\circ}$ grado, oppure nelle (42) è $v=0$.

Nella prima eventualità dalle $D_{1}=D_{2}=\ldots=D_{5}=0$, si trae

$$
\vartheta=\mu \varepsilon \quad, \quad \tau=v \varepsilon,
$$

e ciò porta a concludere che la (42) è riducibile contro l'ipotesi.

Nella seconda eventualità, se $v=0$, dovrà aversi anche $D_{4}=D_{5}=0$, 
ossia $\tau=0$; ed inoltre $D_{1}: D_{2}: D_{3}=1: 2 \mu: \mu^{2}$, il che conduce alla $\vartheta=\mu 3$. Ma allora la (42) è ancora riducibile contro l'jpotesi.

16. b) Caso $R \neq 0$.

Dimostriamo ora il teorema enunciato nel n. 14 nel caso $R \neq 0$. Supponiamo per assurdo non vero il teorema e distinguiamo due sottocasi secondo ohe la funzione $X$ soddisfa ad una equazione differenziale del $1^{\circ}$ ordine di $3^{\circ}$ oppure di $2^{\circ}$ grado in $X, X_{u}, X_{v}$.

$\mathrm{Nel}$ sottocaso 1) le due equazioni (28) e (29) dovranno coincideré. Non potendo essere $S=U=0$, poichè seguirebbe subito $T=0$, supponiamo ad es. $S \neq 0$. Perchè le due equazioni coincidano è intanto necessario che si abbia

$$
T=\mu S \quad, \quad V=\mu^{2} S \quad(\mu \neq 0) .
$$

Dopo aver moltiplicato il $1^{\circ}$ membro della $(28)$ per $\mu$, perchè le equazioni coincidano devono essere identici $i$ polinomi che figurano ai primi membri delle (28) e (29) stesse. Uguagliando $i$ coefficienti di $X X_{u}{ }^{2}, X X_{u} X_{v}, X X_{v}{ }^{2}$, $X^{2} X_{\mu}, X^{2} X_{v}$, si ha uno dei tre casi

$$
\begin{array}{llll}
\mu=2, & 2 \alpha-\delta+9 R=0, & \alpha=0, & \alpha-\delta+3 R=0 \\
\mu=-1, & \alpha+\delta=0 \quad, & \alpha+6 R=0, & \delta+6 R=0 . \\
\mu=\frac{1}{2}, & \alpha-2 \delta-9 R=0, & \alpha-\delta-3 R=0 & \delta=0 .
\end{array}
$$

Da queste segue sempre $R=0$, contro l'ipotesi.

Nel sottocaso 2) si trova che, se esiste un'equazione differenziale del primo ordine di $2^{\circ}$ grado in $X, X_{u}, X_{v}$, questa deve essere necessariamente del tipo

$$
S X_{u}^{2}+2 T X_{u} X_{v}+U X_{v}^{2}+\alpha^{\prime} X X_{u}+\delta^{\prime} X X_{v}+\sigma^{\prime} X^{2}+H_{1}\left(X, X_{u}, X_{v}\right)=0,
$$

dove $S, T, U$ sono gli stessi coefficienti che compaiono nella (20) ed $H_{1}$ è un polinomio di primo grado in $X, X_{u}, X_{v}$.

Se vogliamo che la (44) sia irriducibile non deve essere $U S-T^{2}=0$, poichè ragionando come per il sottocaso 2) del n. precedente, si arriverebbe ad un assurdo.

Se invece è $U S-T^{2} \neq 0$, ragionando come per il sottocaso 1) del n. precedente, devono essere verificate due e solo due delle tre relazioni $\beta=0$, $\gamma=0, \eta=0$. Se ad es. $\beta=\gamma=0$, si deve avere $\alpha^{\prime}=\delta^{\prime}=0$. Poichè d'altra parte si ha

$$
\alpha^{\prime}=\alpha+6 R, \quad \delta^{\prime}=\delta+6 R,
$$

segue ugualmente $\eta_{u}=\eta_{t}=0$ e quindi, $R=0$, contro l'ipotesi. 
17. Determinazione dei tritessuti che ummettono $\infty^{2}$ deformazioni proiettive.

Possiamo ora provare che: $i$ tritessuti che posseggono $\infty^{2}$ deformazioni proiettive sono soltanto $i$ tritessuti $\Delta_{\Sigma}$ e loro eventuali deformati proiettivi di tipo diverso.

Le equazioni (12), per $\lambda=1$, si scrivono

$$
\begin{aligned}
& X_{u u}+2 X_{u v}-3 X\left(X_{u}+2 X_{v}\right)+3 b X_{u}+(6 b+3 \beta) X_{v}+\left(3 a_{u}+6 b_{v}+3 \beta_{v}\right) X=0 \\
& X_{v v}+2 X_{u v}-3 X\left(2 X_{u}+X_{v}\right)+(6 a+3 \gamma) X_{u}+3 a X_{v}+\left(6 a_{u}+3 b_{v}+3 \gamma_{u}\right) X=0 .
\end{aligned}
$$

Sia inoltre

$$
X_{u}=\mu X_{v}+v X+\sigma
$$

l'equazione differenziale del $1^{\circ}$ ordine che, a norma del teorema dimostrato nei nn. precedenti, deve essere compatibile con le (45).

Derivando la (46) ed eliminando poi le derivate seconde, si ottiene uną nuova equazione del $1^{\circ}$ ordine che deve essere identica alla (46). Si ha cosi, in particolare,

$$
(\mu-1)(1+2 \mu)(2+\mu)=0, \quad v\left(1-2 \mu-2 \mu^{2}\right)=0 .
$$

La (47) porge tre possibili valori per la $\mu$ in corrispondenza ai quali deve essere sempre $\nu=0$.

Esaminiarno il caso $\mu=1$ e trascuriamo i casi rimanenti che sono proiettivamente identici a questo.

Perchè l'equazione del $1^{\circ}$ ordine sopra considerata sia identica alla (46) deve aversi inoltre

$$
\begin{aligned}
& \eta=3(b-a)+\beta-\gamma=0 \\
& \sigma=a_{u}-b_{v}-\beta_{v}+\gamma_{u} \\
& \sigma_{u}+\sigma_{v}=3 \sigma(2 a-b+\gamma) .
\end{aligned}
$$

Di queste tre condizioni la (50) è consegnenza delle rimanenti e delle condizioni di integrabilità (5). Di essa non occorrera quindi di tener conto nel seguito. Il sistema di equazioni formato dalle (45), dalla (46) e dalle derivate di questa si scrive

$$
\begin{array}{ll} 
& X_{u}=X_{v}+\sigma \\
& X_{u u}=3 X X_{v}-\xi X_{v}-\chi X-b \sigma+\frac{2}{3} \sigma_{u} \\
& X_{u v}=X_{u u}-\sigma_{u} \\
& X_{v v}=X_{u u}-\sigma_{v}-\sigma_{u}, \\
\text { dove } \quad & \xi=3 b+\beta, \quad \chi=3 b_{v}+2 \beta_{v}-\gamma_{u} .
\end{array}
$$


Perchè il sistema (51) sia chiuso devono ancora sussistere le condizioni

$$
\begin{aligned}
& 3 \sigma-\xi_{u}+\xi_{v}=0 \\
& 3 \sigma_{v}-\chi_{u}+\chi_{v}=0 \\
& 3 \sigma\left(\chi+b_{u}-b_{v}\right)+3 b \sigma_{u}-3 b \sigma_{v}+3 \sigma_{v} \xi+\sigma_{u u}+2 \sigma_{u v}=0 .
\end{aligned}
$$

La (52), tenendo conto della (48), porge

$$
\beta_{v}=\gamma_{u},
$$

mentre la (53) è una consegnenza differenziale della (55).

Ricordando ora che $X=a-\bar{a}=b-\bar{b}$, si ha che gli $\infty^{2}$ tritessuti deformati del dato soddisfano alle condizioni

$$
\begin{aligned}
& \bar{\eta}=3(\bar{b}-\bar{a})+\beta-\gamma=0 \\
& \beta_{v}=\gamma_{u} \\
& \bar{a}_{u}=\bar{b}_{v} .
\end{aligned}
$$

Si vede subito che tali condizioni esprimono appunto che tali tritessuti sono del tipo $\Delta_{\Sigma}$.

La (58) è infatti condizione necessaria e sufficiente affinch ̀̀ la rete costi. tuita dalle due schiere di curve $u=$ cost. $\theta v=$ cost. sia una rete asintotica $\left({ }^{20}\right)$ e cioè una rete ottenuta proiettando da un punto $S$ su un piano $\pi$ le asintotiche di una superficie $F$ (non sviluppabile).

La (57) è condizione necessaria e sufficiente affinchè la superficie $F$ sia proiettivamente deformabile e le curve di $F$ di equazione $d u^{2}-d v^{2}=0$ costituiscano su $F$ una rete $R\left({ }^{21}\right)$.

Infine la (56) ci assicura che la proiezione da $S$ delle curve di $F$ di equazione $d u-d v=0$ sono le sezioni fatte con infiniti piani passanti per $S$.

Ciò è sufficiente per poter affermare che la superficie $F$ e una superficie $\Sigma \theta$ quindi che $i$ tritessuti sono $\Delta_{\Sigma}$. Segue dunque il teorema enunciato al principio di questo n. .

Si tratterebbe ora di vedere se un tritessuto $\Delta_{\Sigma}$ ammette altre deformazioni oltre le $\infty^{2}$, gia indicate al n. 12 , che non lo trasformano in un tritessuto $\Delta_{\Sigma}$. Si puó attraverso un esempio provare che: un generico tritessuto $\Delta_{\Sigma}$ non ammette altre deformazioni oltre le $\infty^{2}$ che lo mutano ancora in un tritessuto $\Delta_{\Sigma}$.

Infatti consideriamo ad es. il tritessuto $\Delta_{\mathrm{X}}$ per cui $\beta=\gamma=1, a=b=1$. L'equazione (20) si scrive in tal caso

$$
X_{u}^{2}-X_{v}^{2}=0
$$

(20) Si reda ad es.: Fubini e CECH, [8], pp. 150 e segg.

(¹) Si veda ad es.: Fubini e $\overline{\mathrm{CECH}}_{\mathrm{E}},[8]$, p. 85. 
Considerando l'equazione $X_{w}-X_{v}=0$, si ottengono le deformazioni di cui al n. 12. Percio le eventuali deformazioni di $\Delta_{\Sigma}$ in un tritessuto di tipo diverso si ottengono con le soluzioni del sistema

$$
\begin{aligned}
& X_{u}+X_{v}=0 \\
& X_{u u}+2 X_{w v}-3 X X_{v}+3 X_{v}=0 \\
& X_{v v}+2 X_{w v}=3 F X_{v}=0 .
\end{aligned}
$$

Ma tale sistema comporta la conseguenza differenziale

$$
X_{v}(X-2)=0
$$

e quindi ammette le sole soluzioni $X=$ cost. che fanno parte delle $\infty^{2}$ che trasformano il tritessuto in un tritessuto $\Delta_{\mathbf{\Sigma}}$.

Non siamo tuttavia in grado di affermare che ogni tritessuto $\Delta_{\mathbf{s}}$ non ammette delle deformazioni che lo trasformano in un tritessuto di tipo diverso.

Osserviamo infine che, siccome le superficie $\mathbf{\Sigma}$ dipendono da quattro funzioni arbitrarie di un argomento, $i$ tritessuti di curve piane deformabili in $\infty^{2}$ modi dipendono da quattro funzioni arbitrarie di una variabile.

\section{I tritessuti che ammettono due famiglie $\infty^{2}$ di deformazioni.}

Esistono particolari tritessuti che ammettono due famiglie $\infty^{2}$ di deformazioni proiettive. Mostreremo in questo n. che:

$i$ tritessuti di curve piane che posseggono due famiglie $\infty^{2}$ di deformazioni proiettive sono $i$ tritessuti $\Delta_{\Sigma}$ provenienti dalle particolari superficie $\Sigma$ rigate caratterizzzate dalle due proprietà :

a) appartengono ad una congruenza lineare generale o speciale;

b) posseggono una rete $R$ di curve appartenenti ai piani di due fasci $i$ cui assi sono due rette incidenti agli assi della congruenza a cui la rigata appartiene.

Consideriamo infatti un tritessuto $\Delta_{\Sigma}$, cioè un tritessuto per il quale siano soddisfatte le condizioni

$$
\eta_{i}=0, \quad a_{u}=b_{v}, \quad \beta_{v}=\gamma_{u} .
$$

Esso possiede allora una famiglia di $\infty^{2}$ deformazioni, rappresentate dal sistema (51) (ove $\sigma=0$ ), che lo mutano in un tritessuto $\Delta_{\Sigma}$. Perchè esso possegga un'altra famiglia $\infty^{2}$ di deformazioni occorre che con le (45) sia com. patibile una delle equazioni

$$
X_{u}+2 X_{v}+h=0, \quad 2 X_{u}+X_{v}+k=0 .
$$

Supponiamo che la prima di tali equazioni sia compatibile con la (45) (il caso rimanente può essere trascurato essendo proiettivamente identico). 
Operando su tale equazione come nel n. precedente si ottengono le condizioni ulteriori

$$
\beta=0, \quad \gamma_{v}=0, \quad h=2 b_{v} .
$$

Essendo $\beta=0$, il tritessuto $\Delta_{\Sigma}$ proviene da una superficie $\Sigma$ rigata. Il sistema di equazioni differenziali ohe rappresentano questa superficie rigata si scrive

$$
\begin{aligned}
& x_{u u}=-b x_{u}+p x \\
& x_{v v}=\gamma x_{u}-a x_{v}+q x,
\end{aligned}
$$

dove, per le (60), (61) e le (5), si ha

$$
\begin{gathered}
a=a(u+v), \quad b=a+\frac{1}{3} \gamma, \quad p=2 b^{2}+a_{u}, \quad q=2 a^{2}+2 b \gamma+a_{v}, \\
\gamma=\text { cost., } \quad 2 a^{\prime}+3 a^{2}+2 a \gamma=k(k=\text { cost. }) .
\end{gathered}
$$

Operando sulle (62) un opportuno cambiamento del tipo $y=\rho x$, si trasformano nelle

dove

$$
\begin{aligned}
& y_{u k}=r y \\
& y_{v o}=\gamma z_{\mu}+s y,
\end{aligned}
$$

$$
r=\frac{3}{4} k+\frac{1}{4} \gamma^{2}, \quad s=r+\frac{1}{4} \gamma^{2} .
$$

Integrando il sistema (63), secondoché : I) $r \neq 0, k \neq 0$; II) $r=0, k \neq 0$; III) $r \neq 0, k=0$, si hanno per la rigata le equazioni parametriche

$$
\begin{aligned}
& x=e^{2 \sqrt{\gamma(u+v)}}, \quad y=e^{2 \sqrt{r u}-r^{v}}, \quad z=e^{(2 \sqrt{r}-\gamma) v} \\
& x=u+v, \quad y=(v-u) e^{-\gamma v}, \quad z=e^{-\gamma v} \\
& x=e^{\gamma(u+v)}, y=e^{\gamma(u-v)}, \quad z=v .
\end{aligned}
$$

Si possono ora verificare direttamente sulle equazioni le proprieta a) e b).

Viceversa se per una rigata sono soddisfatte le proprieta a) e b) appartiene ad uno dei tipi precedenti.

$\mathrm{Si}$ può infine osservare che $\mathrm{i}$ tritessuti deformati dei precedenti sono tutti dello stesso loro tipo. Infatti non esistono altre deformazioni oltre quelle rappresentate dalle (45) e da una delle dae equazioni $X_{\kappa}=X_{v}, X_{u}+2 X_{v}=3 b_{v}$, come mostra l'equazione (20) che nel caso attuale deve necessariamente spezzarsi in queste due ultime equazioni, essendo $R=0$. Inoltre anche i tritessuti della seconda famiglia sono dello stesso tipo dei dati. Infatti essi risultano caratterizzati dalle condizioni

$$
a_{u}+2 b_{v}=0, \quad \eta=0, \quad \beta=0, \quad \gamma_{u}=\gamma_{v}=0,
$$


che mediante il eambiamento di parametri $u=U, v=U+V$ si trasformano nelle $(60),(61)$.

Segue pure che le deformazioni proiettive della seconda famiglia si ottengono facendo il prodotto delle deformazioni della prima famiglia per la deformazione del tritessuto in sè rappresentata dalle equazioni $u=U, v=U+V$.

\section{BIBLIOGRAFIA}

[1] W. BlaschKe e G. BoL, Geometrie der Gewebe, Springer, Berlino (1988).

[2] G. Bor, Ueber Geradengenebe, * Annali di. Matematica * (4), XVII, pp. 45.58 (1988).

[3] E. Bompian, Ise forme elementani e la teoria proiettiva delle superficie, *Boll. Un. Mat. Ital. $",(1), \bar{V}$, pp. 167.173 (1926).

[4] E. Bompiani, Tessuti di curve piane e corrispondenze fra piani, Atti Acc. Naz. Lincei, Rend. ", (8), VI, pp. 7.12 (1919).

[5] BoRÜKa, Sur les correspondences analytiques entre deux plans projectifs, × Pubbl. Univ. Masaryk *, n. 85, pp. 1.34 (1927).

[6] J. Dubourdiwu, Questions topologiques de géométrie différentielle, + Mém, des Soiences Math. ', 78, Paris (1936).

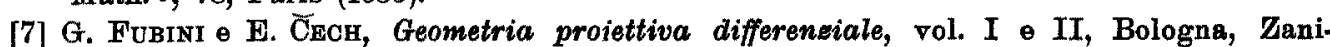
chelli (1926-27).

[8] G. FubINI et E. С̆ Paris, Gathier-Villars (1831).

[9] G. FubinI, Alcuni risultati di geometria proiettivo-differenziale, Atti Acc. Naz. Lincei, Rend. ', (5), XXXII ${ }_{2}$, pp. 273.279 (1928).

[10] G. FubinI, On Abel's converse theorem, *Annals of Math. ', XLIII, pp. 471.:00 (1942).

[11] H. Graf e R. SaUER, Ueber dreifache Geradensysteme in der Ebene, a Munchener Beri. chte , LIV, pp. 119-156 (1924).

[12] H. F. Gronwarr, Sur les équations entre trois variables rappresentables par des nomogrammes à points alignés, -Journ. de Liouville ", (6), VIII (1912).

[13] E. Kollwitz, Projective Invarianten von Kurvengewebe, * Mitteil. Math. Ges, Hamburg *, VII, pp. $132-147$ (1933).

[14] L. Muracchivi, Trasformazioni puntuali e loro curve caratteristiche, Atti IV Congresso Un. Mat. Ital. Taormina 1951 \$, pp. 417.424, Roma, Perrella, (1953).

[15] L. MURAcchins, Contributo alla geometria proiettiva differenziale dei 3-tessuti di curve piane, Boll. Un. Mat. Ital. s, (3), VII, pp. 285.292 (1952).

16] L. Muracchin, Osservazioni sull'applicabilitò proiettiva di due trasformazioni puntuali fra piani, ‘Boll. Un. Mat. Ital. ’, (3), XII, pp. 176-182 (1957).

[17] B. SEGRE, Intorno alla teoria delle superficie proiettivamente deformabili e alle equazioni differenziali ad esse collegate, “Mem. della Reale Acc. d'Italia ", II, n. 3, pp. 1-143 (1931).

[18] G. Thomsen, Lin teorema topologico sulle schiere di curve e una caratterizzazione geometrica delle superficie isotermo-asintotiche, * Boll. Un. Mat. Ital. ", (1), VI, pp. 80-85 (1927).

[19] M. VILLA e L. MURacchini, L'applicabilità proietiva di due trasformazioni puntuali, - Boll. Un. Mat. Ital. ", (3), X, pp. 313.327 (1955). 